\title{
Electrical Properties of Polypyrrole Conducting Polymer at Various Dopant Concentrations
}

\author{
N. Othman ${ }^{1}$, Z. A. Talib ${ }^{1, *}$, A. Kassim ${ }^{2}$, A. H Shaari ${ }^{1}$ and J.Y.C. Liew ${ }^{1}$ \\ ${ }^{1}$ Department of Physics, Faculty of Science, Universiti Putra Malaysia, 43400 UPM Serdang, Selangor. \\ ${ }^{2}$ Department of Chemistry, Faculty of Science, Universiti Putra Malaysia, 43400 UPM Serdang, Selangor. \\ *Author to whom correspondence should be addressed; E-mail: zainalat@science.upm.edu.my
}

Received: 31 October 2008, Revised: 13 January 2009

Online Publication: 27 May 2009

http://dx.doi.org/10.11113/mjfas.v5n1.284

\section{ABSTRACT}

Polypyrrole conducting polymer was prepared by chemical reaction method with various concentrations of iron (III) chloride $\left(\mathrm{FeCl}_{3}\right)$ as dopant. The dc conductivity was obtained from current-voltage characteristic by using parallel-plate techniques in the temperature range of $100-300 \mathrm{~K}$. With the involvement of chloride, $\mathrm{Cl}^{-}$in the polymeric chain, the conductivity increased as temperature and the dopant concentration increased. To describe the electrical transport process, Mott's 1-D, 2-D and 3-D variable range hopping (VRH) models have been considered. The result gives evidence of transport mechanism based on Mott's 3-D VRH mechanism for all various dopant concentrations studied.

| Conducting polymer | DC conductivity | Mechanism |

\section{Introduction}

Generally, polymers are insulators. In view of their properties they are considered as uninteresting and have limited applications from the point of view of electronic materials. However, in 1970s, the first polymer capable of conducting electricity, polyacetylene doped with iodine was reported [1]. The success proved that the polymer conductivity can be increased to as high as 15 orders of magnitude by oxidative doping effects [2]. Its simple synthesis process [3], used as coatings for corrosion protection [4], capable of exhibiting a significant level of electrical conductivity [5] and have good environmental stability in the presence of oxygen and water [6,7] has also given conducting polymer advantage over metal.

Conducting polymers such as polyaniline, polythiopene and their derivatives, polypyrrole (PPy) are among the best candidate materials used in commercial applications such as in solar cells, electromagnetic shielding, electrode for rechargeable batteries, sensors, supercapacitors and corrosion protection [8]. The PPy conducting polymer is processable when doping with either inorganic or organic acid with various methods such as chemical and electrochemical methods [9]. During the polymerization process, the dopant leads to the formation of conjugation defects along backbone of the polymer chain. The defect involves rearrangement of the double bond in the conjugated system and provides a highway for charge carrier to move along the chain. The mechanism can be explained by quasi-particle such as polarons and bipolarons in $\pi$-bonds of the conjugated system. 
The early point of view on the nature and mechanism of transport in conducting polymers are similar to amorphous semiconductor [10]. According to this analogy the conduction mechanism of thermal activation, Mott's 1-D, 2-D and 3-D variable range hopping (VRH) models have been proposed [11]. To have an overview of the mechanism in doped PPy conducting polymer we present its dc conductivity behaviour as a function of temperature with various dopant concentrations.

\section{Experimental Method}

The PPy conducting polymer was prepared by chemical reaction method. The monomer pyrrole (Fluka) was distilled at temperature $131^{\circ} \mathrm{C}$ and stored at $4{ }^{\circ} \mathrm{C}$ before use. A mixture solution containing pyrrole of 0.2 mole concentration and $\mathrm{FeCl}_{3}$ solution (HmBG Chemicals) with different mole concentrations resulting in 0.5 to 3.0 molar ratio, MR (oxidant / monomer). During the mixing process, the solution colour changed from transparent to dark green, indicating that the polymerization takes place instantaneously. The mixture was stirred for 6 hours at ambient temperature. The resulting powder were then filtered and washed with distilled water to remove the adhered $\mathrm{Fe}^{3+}$ and dried at $40^{\circ} \mathrm{C}$ for 48 hours. The fine powder was then pressed into pellet at 7 ton $/ \mathrm{cm}^{2}$ pressure. The pellets were then coated with silver paint and dried at $50^{\circ} \mathrm{C}$ before taking any electrical measurement. The electrical conductivity was measured using parallel-plate technique. Source Measurement Unit Keithley 236 was used to supply the constant current and measure the resulting voltage in temperature range of 100 to $300 \mathrm{~K}$.

\section{Results and Discussions}

The conductivity $(\sigma)$ dependence on PPy conducting polymer doped with $\mathrm{FeCl}_{3}$ at various molar ratio, $\mathrm{MR}$ at room temperature is depicted in Figure 1(a). The plots show that the conductivity increases with increases in MR. It shows the conductivity change from $8 \times 10^{-2}$ to $2 \times 10^{-1} \mathrm{~S} / \mathrm{cm}$. This indicates that in the PPy chain, amino<smiles>CCCC1NC(C)CC1NCC</smiles>

groups in the pyrrole rings ( $\mathrm{H}_{\mathrm{H}}$ ) donates $\pi$-electron to the aromatic network of the polymer and make themselves become less electron. When $\mathrm{FeCl}_{3}$ was added into the polymer, it forms a donor-acceptor complex in the conjugated system. As a result, quasi-particle are created presumably polarons and acts as a charge carriers. At low dopant concentration the polarons have low mobility and exhibit low conductivity. When the polymerization proceeds with increased doping level, more $\mathrm{Cl}^{-}$is bound to the pyrrole ring in the backbone of the polymer chain. At this point, many polarons are created and become crowded to have enough energy to form bipolarons. This increases the mobility of the charge carriers which causes the increase in conductivity.

To learn more about the mechanism of electrical conductivity, the functional dependence of the conductivity on temperature must be determined. Figure 1(b) can be used for preliminary characterizations. It was seen that the conductivity increased with temperatures. This can simply be associated to the contribution of the charge carrier mobility. In this case, the polarons or bipolarons might move with higher diffusion velocity when the temperature increased and thus increased the conductivity [12]. In order to get more insight into the transport mechanism, various models were used to fit the data from Figure 1(b). The models for thermally activated and Mott's variable range hopping (VRH) is given by Equations (1) and (2) respectively:

$$
\begin{gathered}
\sigma=\sigma_{o} \exp \left(-\frac{E_{a}}{k_{B} T}\right) \\
\sigma=\sigma_{o} \exp \left\{-\left(\frac{T_{o}}{T}\right)^{1 /(1+d)}\right\}
\end{gathered}
$$


where $\sigma$ is the conductivity, $\sigma_{o}$ is a constant, $E_{a}$ is the activation energy, $k_{B}$ is the Boltzmann constant (8.617 $\left.343 \times 10^{-5} \mathrm{eV} / \mathrm{K}\right), T$ is the temperature, $d=1,2,3$ is the dimensionality of the conduction process and $T_{o}$ is associated with the degree of localization of the electronic wave function.

Figure 2(a) shows that the conductivity value when plotted as Log $\sigma$ versus 1000/T does not exhibits linear dependency but markedly curved which indicates that the conductivity does not correspond to a thermally activated mechanism. It has been shown that, in low temperature range the charge carrier are electrostatically bound to the $\mathrm{Cl}^{-}$and therefore they are not expected to move [12]. For this reason, the Equation (2) was considered where the information on the conduction mechanism and the dimensionality of the process can be determined. The mechanism described the Mott's variable range hopping (VRH) model which describes the transport by hopping of states near Fermi energy, $E_{f}$. These states are located at specific states in the energy gap and could act as hopping centers. The hopping probability does not only depend on the energy difference between these localized states but also on the overlap of the electron wave function [13].

The plots of $\log \sigma$ versus $\mathrm{T}^{1 /(1+\mathrm{d})}$ in Figure 2(b), (c) and (d) show that $\mathrm{d}=1,2$ and 3 all give straight line curve. However, $\mathrm{d}=3$ gives a better linear regression value, $\mathrm{R}^{2}$ which indicate the best fit between the 3 curves. This point indicating that the transport mechanism in PPy conducting polymer correspond to a 3-dimensional, 3-D process. The values of the linear regression are calculated for different mechanisms as given in Table 1.
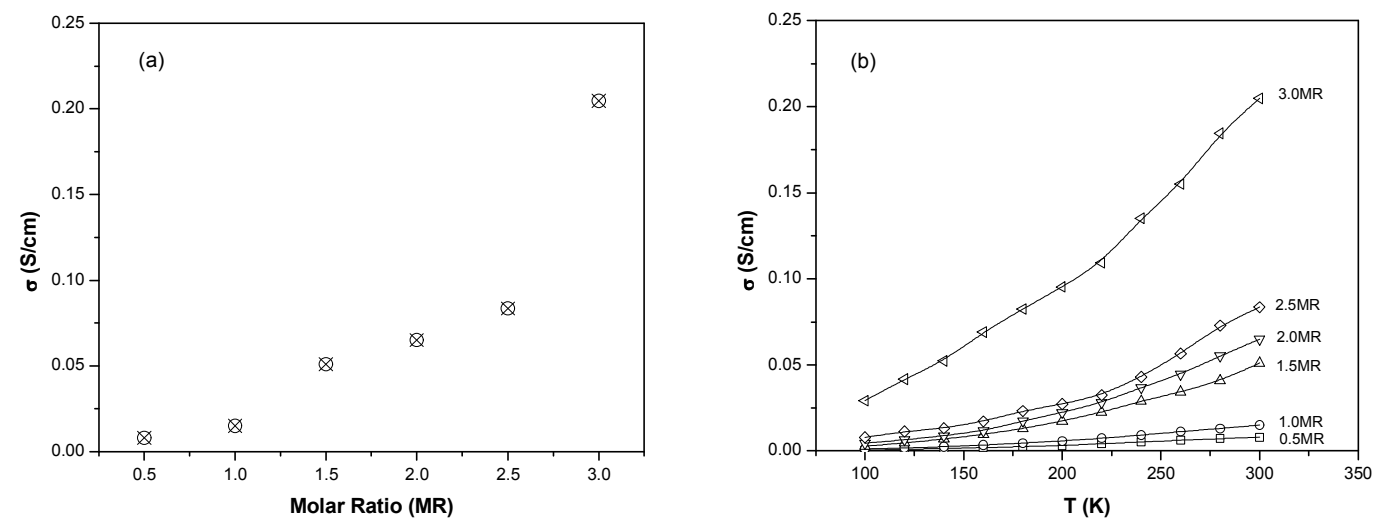

Figure 1: Plots of conductivity, $\sigma$ as functions of (a) molar ratio $\left(\mathrm{FeCl}_{3} /\right.$ monomer of pyrrole) and (b) temperatures ranging from $100-300 \mathrm{~K}$.

\section{Conclusions}

Bulk samples of PPy conducting polymer was successfully prepared by chemical polymerization at various dopant concentrations and their electrical transport properties were studied by using parallel-plate technique. It was observed that the electrical conductivity in the temperature range 100 to $300 \mathrm{~K}$ increases with increase dopant concentrations and temperature. The fitting of the experimental temperature dependence data reveals that the Mott's 3-D variable range hopping mechanism is the most suitable mechanism for PPy conducting polymer behavior. 

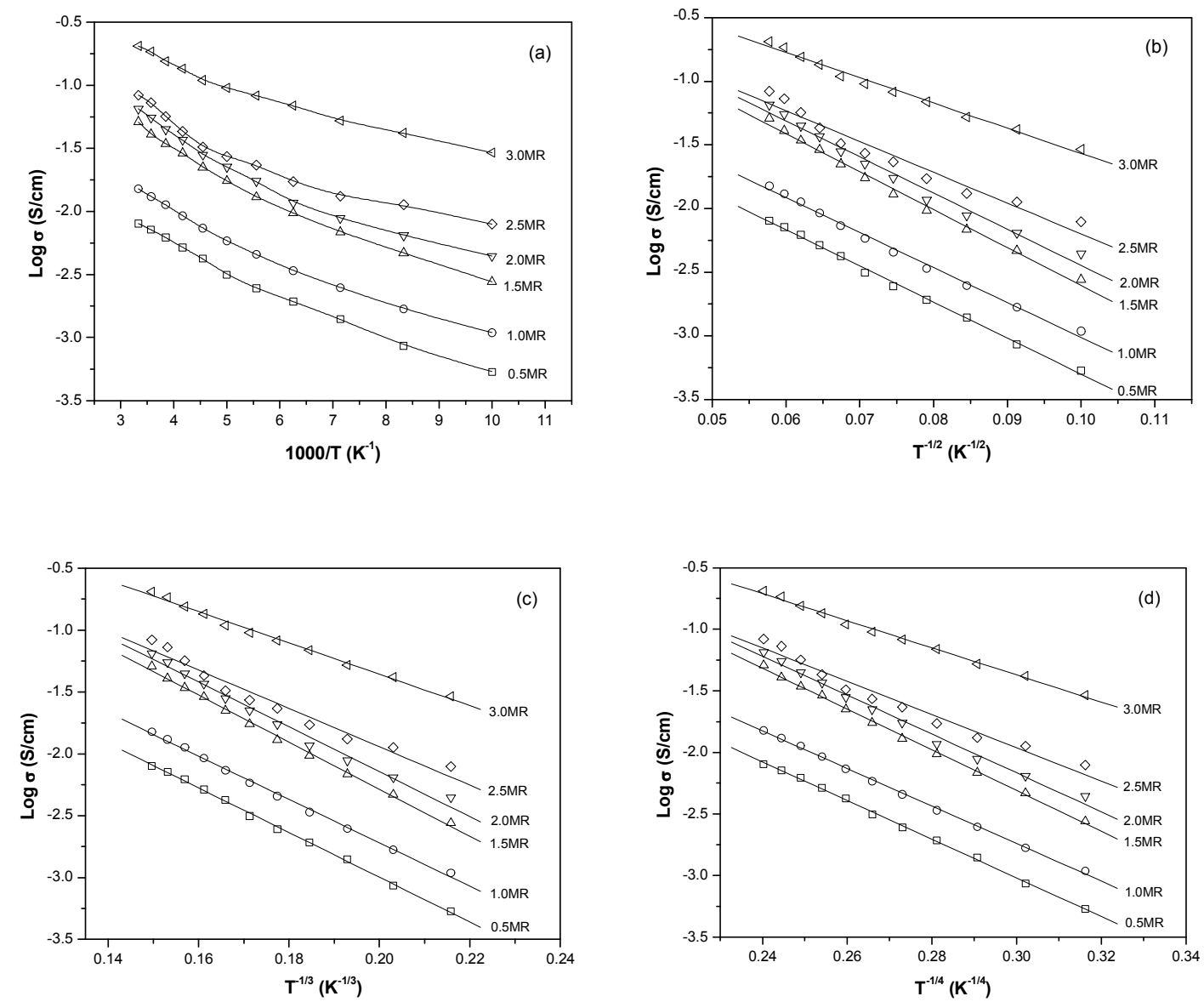

Figure 2: Plots of $\log \sigma$ as functions of (a) $1000 / \mathrm{T}$, (b) $\mathrm{T}^{-1 / 2}$, (c) $\mathrm{T}^{-1 / 3}$ and (d) $\mathrm{T}^{-1 / 4}$.

Table 1: Calculated values of linear regression with different mechanisms.

\begin{tabular}{|l|c|c|c|c|c|c|}
\hline & \multicolumn{7}{|c|}{ Linear Regression } \\
\hline Transport mechanism & $\mathbf{0 . 5 M R}$ & $\mathbf{1 . 0 M R}$ & $\mathbf{1 . 5 M R}$ & $\mathbf{2 . 0 M R}$ & $\mathbf{2 . 5 M R}$ & $\mathbf{3 . 0 M R}$ \\
\hline Thermal activation & 0.99295 & 0.98863 & 0.98827 & 0.97822 & 0.95829 & 0.98471 \\
\hline Mott's 1D-VRH & 0.99875 & 0.99727 & 0.997 & 0.99122 & 0.97672 & 0.9946 \\
\hline Mott's 2D-VRH & 0.99935 & 0.99881 & 0.99861 & 0.99424 & 0.98168 & 0.99665 \\
\hline Mott's 3D-VRH & 0.99939 & 0.99933 & 0.99915 & 0.99549 & 0.98392 & 0.99742 \\
\hline
\end{tabular}




\section{Acknowledgement}

The authors would like to acknowledge the financial assistance given by Ministry of Science Technology and Foundation and Ministry of Education through e-Science Fund (5450040) and Fundamental Science Research Grant (5523123) and scholarships from National Science Foundation.

\section{References}

[1] Alan J.Heeger, Nobel Lecture, (2000).

[2] Malhotra B.D. and Singhal Rahul, Indian Academy of Sciences 61, (2003), 331-343.

[3] Lin-Xia Wang, Xin-Gui Li and Yu-Liang Yang, Reactive \& Functional Polymers 47, (2001) 125-139.

[4] Michael Rohwerder and Adam Michalik, Electrochimica Acta 53 (2007) 1300-1313.

[5] H. N. M. Ekramul Mahmud, Anuar Kassim, Zulkarnain Zainal and Wan Mahmood Mat Yunus ScienceAsia 31 (2005) 313-317.

[6] D. Kumar and R.C Sharma, Eur. Polym. J. 34 (1998) 1053-1060.

[7] S.H. Hong, B.H. Kim, J. Joo, J.W. Kim and Hyung J. Choi, Current Applied Physics 1 (2001) 447-450.

[8] Amarjeet K Narula, Ramadhar Singh and Subhas Chandra, Indian Academy of Sciences 23 (2000) 227-232.

[9] M. Yamaura, T. Hagiwara and K. Iwata, Synthetic Metals, 26(1988) 209 - 224.

[10] Epstein A.J, Lee W.P. and Prigodin V.N., Synthetic Metals, 117 (2001) 9-13.

[11] Singh Ramadhar, Kumar Jitendra, K. Singh Rajiv and Rastogi C. Ramesh, New J. Phys. 9 (2007) 40.

[12] Siegmar Roth, Material Science Forum, 21 (1987) 1-12.

[13] Terje A. Skothem, Handbook of Conducting Polymers, Vol.2, New York, (1986). 